\title{
Role of topical cholecalciferol granules for antimicrobial drug delivery in diabetic foot ulcers
}

\author{
Chander Shekhar $^{1}$, Abhinav Aggarwal ${ }^{2}$, Ravi Kumar Chittoria ${ }^{3 *}$, Praveen Upadhyay ${ }^{4}$, Vinayak Chavan $^{5}$, \\ Saurabh Gupta ${ }^{6}$
}

${ }^{1}$ Consultant, ${ }^{2,5,6}$ Senior Resident, ${ }^{3}$ Professor and Registrar, ${ }^{4}$ Junior Resident, ${ }^{1}$ Dept. of Orthopaedics, ${ }^{2,3,5,6}$ Dept. of Plastic Surgery, ${ }^{4}$ Dept. of General Surgery, ${ }^{1}$ Apollo Hospital, New Delhi, ${ }^{2-6}$ Jawaharlal Institute of Post Graduate Medical Education and Research (JIPMER), Pondicherry, India

\section{*Corresponding Author: Ravi Kumar Chittoria}

Email: abhi2128@gmail.com

\begin{abstract}
Diabetic mellitus, a metabolic disorder, has a high incidence and morbidity. Of all complications, diabetic foot ulcer is one of the commonest with $25 \%$ likelihood of a diabetic to develop diabetic for ulcers. Infection in a diabetic foot ulcer is a dreaded complication with often complicates the clinical course of the patient and is managed by local and systemic antibiotics. Local antibiotics are generally deemed ineffective in various studies due to lack of an appropriate drug delivery system.

The Vit D level is inversely related to development of diabetic vascular disease. A novel strategy for treating diabetic foot ulcer with osteomyelitis may be using topical antibiotics with drug delivery system which will have minimum systemic side effects.

Our case, a middle age male, having right foot diabetic ulcer with (Wagner stage 2) uncontrolled sugar levels. A trial of topical antibiotic therapy with tobramycin using cholecalciferol granules as a drug delivery system was given. Regular dressing was continued and serial wound cultures were taken.

The wound culture was became sterile after 4 application of the therapy (14 days) with cholecalciferol granules with healthy granulation. The wound was fit for reconstruction after 5 applications.

This report is an attempt to bring to the notice that such a drug delivery system is efficacious and may be validated using a larger trial.
\end{abstract}

Keywords: Topical cholecalciferol, Antimicrobial drug delivery, Diabetic foot ulcer.

\section{Introduction}

Current burden of diabetes mellitus in the world is estimated to be around 415 million adults. Treating diabetes accounts for $12 \%$ of the entire healthcare expenditure in the world. The estimated lifetime risk for a diabetic to develop a foot ulcer is estimated to be around 25\%. Diabetic foot is one of the most devastating complication of diabetes which is having high socioeconomic burden of around 10,000 EUR on an average for a non-infected ulcer. About $15-25 \%$ of patients with diabetes have the possibility of developing foot ulcer during their life. Among the all diabetic patients the prevalence of diabetic foot osteomyelitis occurs in 2$3 \% .{ }^{1}$ Diabetic foot osteomyelitis results poor life quality due to its characteristic chronicity.

Pathophysiology of diabetic foot ulcers has been bee well described and explained due to peripheral neuropathy, peripheral arterial disease and a blunted immune response. The chances of these ulcers to become infected are particularly high and statistically, one individual loses a limb to an infected diabetic foot ulcer every 20 seconds.

Infection in diabetic foot ulcers is described as having one of the two classical signs and symptoms of inflammation (Pain, tenderness, warmth, redness, swelling) or purulent discharge. Foot problems are associated with more hospitalisation in diabetes than any other complication. $^{2}$

The treatment options for Diabetic foot ulcers includes, debridement, management of wound infection by iv or oral antibiotics and off load dressing of the ulcer, negative pressure wound therapy and hyperbaric oxygen. The most important measures for diabetic foot osteomyelitis is intravenous antibiotics. Intravenous antibiotics have demerits of causing systemic toxicity. One novel idea is using topical antibiotics to overcome the systemic toxicity. ${ }^{3}$

VIT D, that is cholecalciferol, causes increase in calcium absorption and causes increased level of calcium level in plasma. Several studies has reported that Cholecalciferol have additional anti-inflammatory property, moreover evidence from various epidemiological study has shown inverse relationship between the level of Cholecalciferol level and development of diabetic vascular diseases. ${ }^{4}$

Topical antibiotic delivery systems are an area of controversy with no clear consensus. The lack of published literature on this topic may be, in part, due to difficulty in conducting such studies in patients having multiple comorbidities and having complex chronic wounds. ${ }^{4}$

We here present a case of diabetic foot ulcer of 6 month duration, managed with a novel topical antibiotic drug delivery system.

\section{Case Report}

38 year old male, a manual labour, presented with a complaint of an ulcer on right foot heel over 6 months. Had initially a small blister on the heel which burst spontaneously. He was a known diabetic, diagnosed 6 months ago, on oral hypoglycaemic drugs with uncontrolled sugars. The wound was involving the heel pad and had near total complete loss of heel pad. It was diagnosed to be Wagner Stage 2 clinically (Fig. 1). X ray was not suggestive of osteomyelitis. 
The patient was immediately admitted, worked up and immediate debridement was done in the emergency OT and broad spectrum antibiotics were started (Fig. 2). Wound tissue culture was sent and it came out to be acinetobacter baumannii and Enterobacter resistant to all antibiotics. $\mathrm{HbA1C}$ came out to $14.7 \mathrm{gm} / \mathrm{dl}$ and his sugars were not controlled. Insulin was started and titrated according to the sugars.

Patient had raised counts and deranged renal functions. Decision was made to give a trial of topical antibiotic therapy with tobramycin mixed with cholecalciferol granules after due consent of the patient.

One gram sachet $(60,000$ IU per gram $)$ of cholecalciferol granules is mixed with one $\mathrm{ml}(40 \mathrm{ml})$ of Tobramycin (Fig. 3) and thick paste is made. The paster is applied directly over the wound with a sterile applicator (Fig. 4). This is covered with standard dressing of gauze and pad. Repeat dressings were done after every 3 days and repeat tissue culture was taken at the time of each dressing. The Wound tissue culture became sterile after 4 applications and the wound also developed healthy granulation (Fig. 5). The wound improved after just 5 changes of cholecalciferol and Tobramycin dressing and was deemed fit for reconstruction.

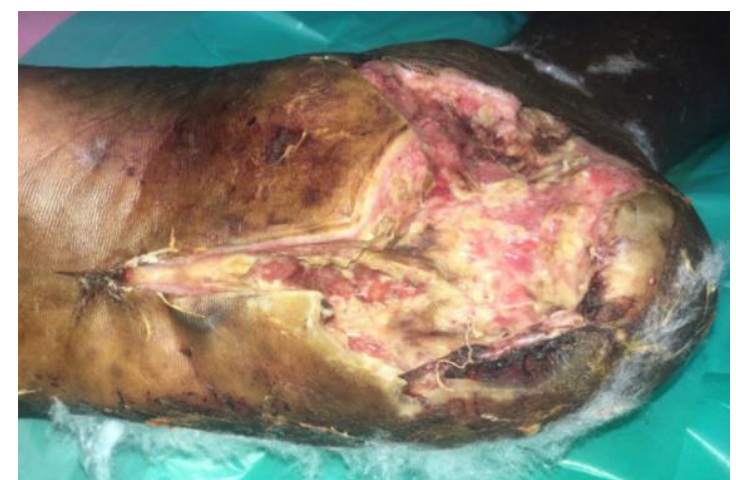

Fig. 1: Wound on presentation

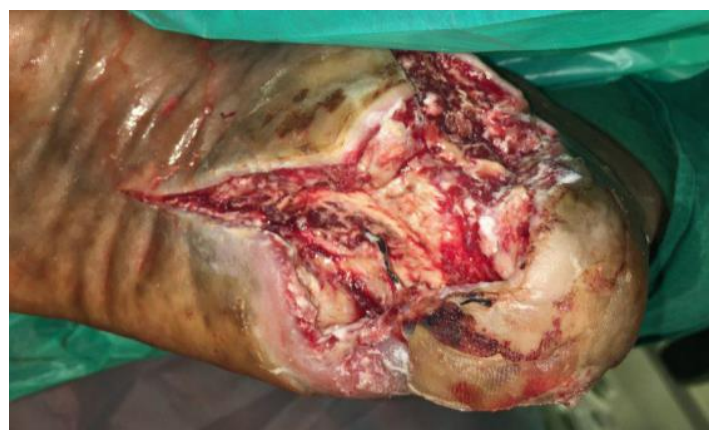

Fig. 2: Wound after debridement

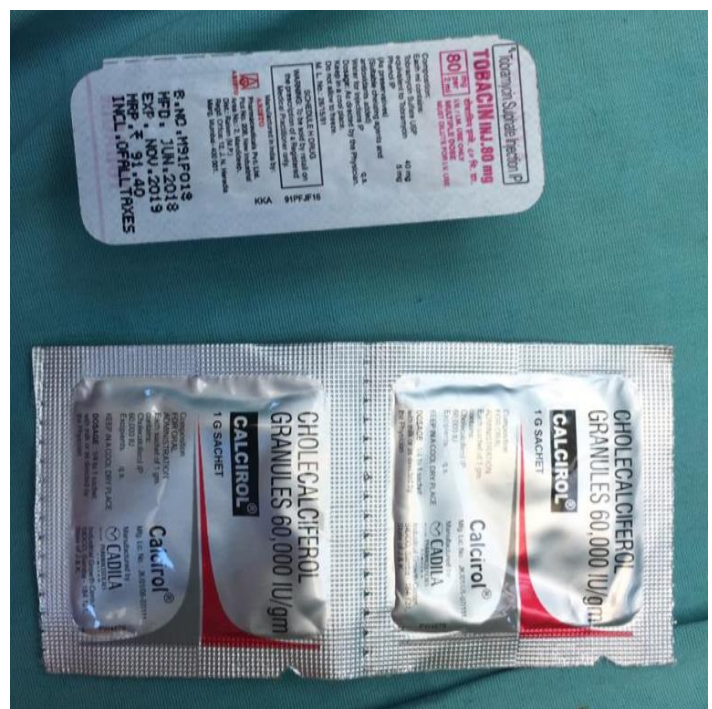

Fig. 3: Cholecalciferol and tobramycin ampoule used

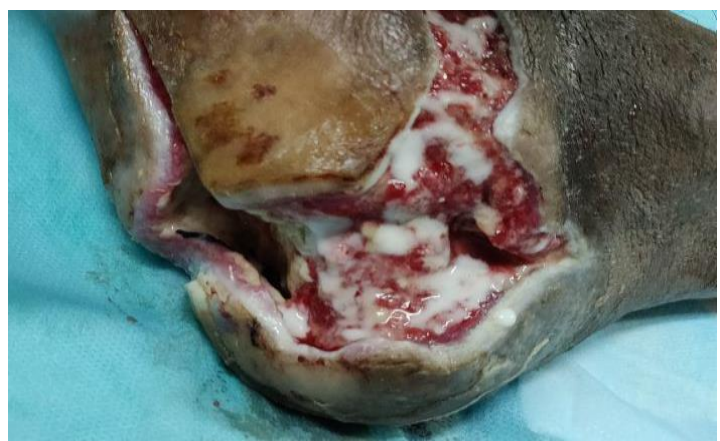

Fig. 4: Paste applied to the wound

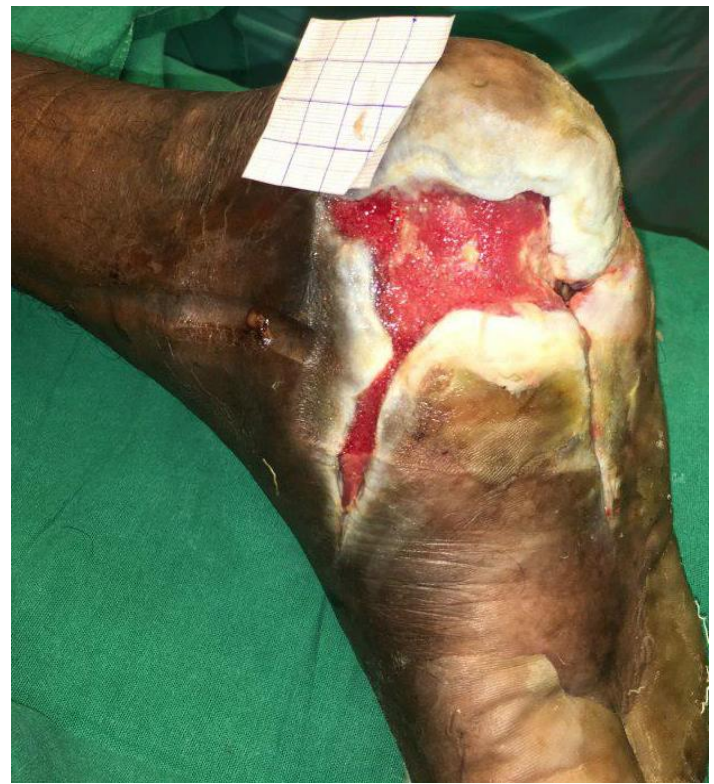

Fig. 5: Wound after 5 applications

Discussion

Use of Cholecalciferol as a drug delivery method for antibiotics is a novel idea. 
It has immunomodulatory properties are well known and it also induces the expression of innate antimicrobial peptides. It reduces inflammation and improves immunity. It has been reported to improve corneal wound healing in a study by reins et al in $2015 .^{5}$

Yakob et al found that the low levels of vitamin D could be related in the development of diabetic foot infections. A few in vitro studies demonstrated that cholecalciferol may lead to production of anti microbial peptides in cells of diabetic foot ulcer and thus can hasten the process of wound healing. The topical applications of Vitamin D in wound has been studied in rats, and it was reported that topical application of Vitamin D accelerates wound healing in dose dependent manner. ${ }^{6}$

Study on topical use of Vitamin D in healing of Diabetic foot and diabetic foot osteomyelitis in human has not been done extensively and there is a need for further study to establish a definitive role of topical Vitamin D in the treatment of diabetic foot ulcer.

Treatment of an infection requires antimicrobial therapy, which may be given systemically through oral or intravenous or intramuscular route or locally through application of antiseptic, antibiotic, or other antimicrobial preparations. A recent cochrane review assessed all the available evidence on use of topical antimicrobial agents for preventing or treating infection in diabetic foot ulcers. The review included studies evaluating treatment with any type of solid (liquid, gel, ointment, cream) topical antimicrobial agent, including antiseptics and antibiotics. The review concluded that the randomized controlled trial data on the effectiveness and safety of topical antimicrobial treatments for diabetic foot ulcers is limited by the availability of relatively few, mostly small, and often poorly designed trials. Based on this review the authors recommended that 1) use of an antimicrobial dressing instead of a nonantimicrobial dressing may increase the number of diabetic foot ulcers healed over a medium-term follow-up period (low-certainty evidence); and 2) there is probably little difference in the risk of adverse events related to treatment between systemic antibiotics and topical antimicrobial treatments. ${ }^{2}$

The idea of using topical antibiotic therapy over systemic antibiotic therapy is based on the presumption that the systemic side effects will be minimised and lead to better bioavailability at the required site. Moreover, its an indigenous idea with extremely low cost to the health care provider. The total cost of one dressing with cholecalciferol granules and one ampoule tobramycin was USD 2 to $3 \$$ which is extremely cost effective with great results.

The authors have tried the similar therapy for various similar chromic wounds with astounding results.

It leads to control of the biofilm production, improvement in wound environment and elimination of bacterial load.

A similar large multi centre trial is being planned to understand and validate the results.

\section{Acknowledgments}

None.

\section{Financial Support and Sponsorship: None.}

\section{Conflicts of Interest: None.}

\section{References}

1. Ogurtsova K, da Rocha Fernandes JD, Huang Y, Linnenkamp U, Guariguata L, Cho NH, Cavan D, Shaw JE, Makaroff LE. IDF Diabetes Atlas: Global estimates for the prevalence of diabetes for 2015 and 2040. Diabetes research and clinical practice. 2017;128:40-50.

2. Dumville JC, Lipsky BA, Hoey C, Cruciani M, Fiscon M, Xia J. Topical antimicrobial agents for treating foot ulcers in people with diabetes. Cochrane Database Syst Rev 2017(6).

3. Jeffcoate WJ, Harding KG. Diabetic foot ulcers. Lancet 2003;361(9368):1545-51.

4. Cowling T, Jones S. Topical Antibiotics for Infected Wounds: A Review of the Clinical Effectiveness and Guidelines. 2017

5. Reins RY, Hanlon SD, Magadi S, McDermott AM. Effects of topically applied vitamin D during corneal wound healing. PloS one 2016;11(4):e0152889.

6. Yakob, M. A. H., Leong, J. F. \& Pande, K. C. Vitamin D and other biochemical markers of nutrition in patients with diabetic foot infection in negara brunei darussalam. In IOF Regionals5th Asia-Pacific Osteoporosis Meeting. Osteoporos Int 2014;25:639.

How to cite this article: Shekhar C, Aggarwal A, Chittoria RK, Upadhyay Pn, Chavan V, Gupta S. Role of topical cholecalciferol granules for antimicrobial drug delivery in diabetic foot ulcers. Indian J Orthop Surg 2019;5(2):165-7. 